Presented at PST 2003 Workshop on Polarized Sources and Targets

Novosibirsk, Russia Sept. 22-26, 2003

BNL-72314-2004-CP

\title{
ABSOLUTE POLARIZED H-JET POLARIMETER DEVELOPMENT FOR RHIC
}

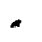 \\ $\underline{\text { A.Zelenski }^{1}}$, A.Bravar ${ }^{1}$,D.Graham ${ }^{1}$, W.Haeberli ${ }^{2}$, S.Kokhanovski ${ }^{3}$, \\ Y.Makdisi ${ }^{1}$, G.Mahler ${ }^{1}$, A.Nass ${ }^{1}$, J.Ritter ${ }^{1}$, T.Wise ${ }^{2}$, V.Zubets ${ }^{3}$ \\ 1-Brookhaven National Laboratory, 2-University.of Wisconsin-Madison, 3-INR, Moscow

\begin{abstract}
Absract. Status of the.H-jet polarimeter development is reviewed. The preliminary results of atomic beam intensity and density measurements are presented.
\end{abstract}

\section{INTRODUCTION}

A polarized $\mathrm{H}$-jet polarimeter was proposed for the absolute polarization measurements in RHIC /1-4/. It is based on elastic proton-proton scattering in the Coulomb-Nuclear Interference (CNI) region. Due to particle identity, polarization of the accelerated proton beam can be directly expressed in terms of proton target polarization, which can be precisely measured by a Breit-Rabi polarimeter $/ 5 /$. These measurements will be used for calibration of a fast $\mathrm{p}$-Carbon CNI polarimeter, which has proven to be a very effective instrument for depolarization studies and polarization time-evolution monitoring during acceleration and storage in RHIC /6/. An accuracy of about $2 \%$ for the CNI elastic p-p collision analyzing power is expected from the measurements with the polarized H-jet in RHIC, which will allow to determine the hadronic spin-flip contribution to CNI process (at 100-250 GeV energy) also to about $2 \%$ accuracy, in comparison with $20 \%$ accuracy from E-704 experiment at FNAL /2/.

The polarimeter target is a free atomic beam, which crosses the RHIC beam in the vertical direction. With a state-of-art polarized atomic beam source, the $\mathrm{H}$-jet target thickness of about $10^{12}$ atoms $/ \mathrm{cm}^{2}$ was expected $/ 3 /$. The measured H-jet thickness at the collision region (as seen by stored high-energy beam) is consistent with the calculations. This will provide a polarimeter counting rate of about 100 events/s for the 2004 run. At $100 \mathrm{~Hz}$ event rate the proton beam polarization can be measured to $\sim 2 \%$ statistical accuracy in $20 \mathrm{hrs}$ integration time. It is estimated, that systematic errors will limit the total accuracy to about $10 \%$ (the goal for the first run) and eventually to $5 \%$ absolute polarization measurements accuracy. The $\mathrm{H}$-jet polarimeter will be installed at the RHIC beams intersection IP-12, which is presently not occupied. Due to the small $\mathrm{H}$-jet target thickness $\left(\sim 10^{12}\right.$ atoms $\left./ \mathrm{cm}^{2}\right)$, the polarimeter can be operated continuously, without any effect on the RHIC beams, and the remote location excludes any background generation for the other experiments.

*This work was performed under the auspices of U.S.D.O.E., Contract No. DE-AC02-98CH10886. 


\section{EXPERIMENTAL SETUP}

Polarimeter vacuum system: The H-jet polarimeter includes three major parts: polarized Atomic Beam Source (ABS), scattering chamber, and Breit-Rabi polarimeter (BRP) (see Fig.1). The polarimeter axis is vertical and the recoil protons are detected in the horizontal plane. The common vacuum system is assembled from nine identical vacuum chambers, which provide nine stages of differential pumping. The system building block is a cylindrical vacuum chamber $50 \mathrm{~cm}$ in diameter and $32 \mathrm{~cm}$ long with four $20 \mathrm{~cm}(8.0$ ") $\mathrm{ID}$ pumping ports. Each chamber is pumped by two turbomolecular pumps (TMP) with ceramic bearings. A third pump was installed at the first dissociator stage, which resulted in 5\% beam intensity increase. The TMP features high $\sim 10^{6}$ compression ratio for $\mathrm{H}_{2}$ pumping and about $1000 \mathrm{l} / \mathrm{sec}$ pumping speed.

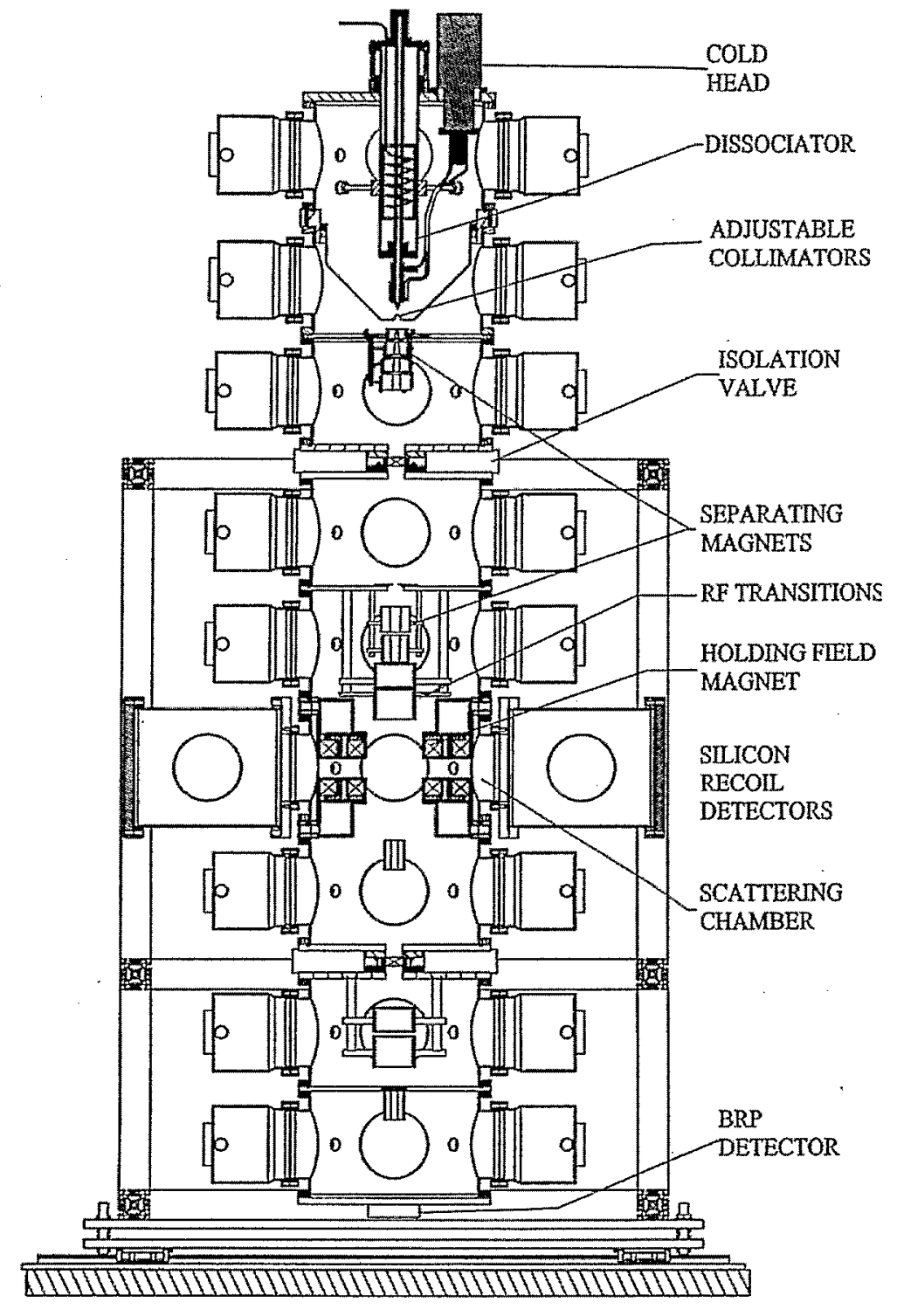

Figure 1: H-jet polarimeter general layout. 


\section{a). POLARIZED ATOMIC BEAM SOURCE.}

The ABS part of the H-jet polarimeter includes five vacuum chamber and five differential vacuum stages (see Fig.1). The distance from the nozzle to collision point is $127 \mathrm{~cm}$.

Dissociator. In conventional dissociator design a pyrex or quartz tube is cooled by water jacket and only aluminum nozzle at the end of the tube is cooled to $30-100^{\circ} \mathrm{K}$ to produce a low velocity atomic beam. The subsequent high thermal gradient may cause insufficient atomic hydrogen gas cooling. In the dissociator design described here we took an advantage of use a high-power Leybold-120RGS cryocooler, which produces about $30 \mathrm{~W}$ cooling power at $40^{\circ} \mathrm{K}$ operational temperature. The power excess is used for cooling of the last $12 \mathrm{~cm}$ of the dissociator ("dissociator neck") (see Fig.2) to provide an extended thermal gradient region between the end of the water jacket and the nozzle.

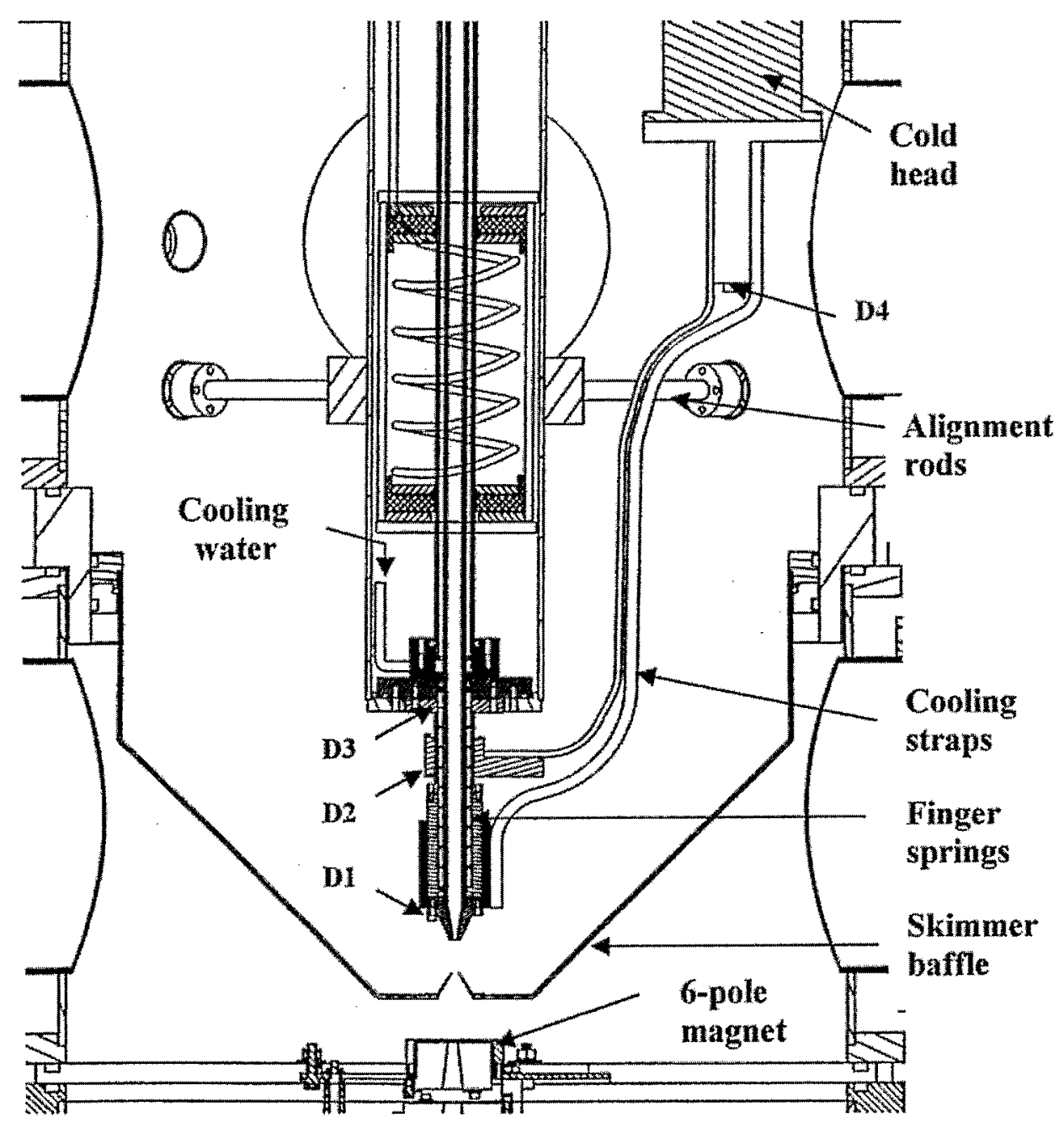

Fig.2. The dissociator and adjustable skimmer assembly. D1-D5-silicon diode temperature sensors. 
Two flexible copper cooling straps provide heat conductance between cold head and the dissociator neck. The nozzle strap consists of 30 copper foils $(0.3 \mathrm{~mm}$ thick, $50 \mathrm{~mm}$ wide and about $380 \mathrm{~mm}$ long). The strap is clamped to a heavy wall copper tube (see Fig.2). The heat conductance between copper tube and quartz dissociator liner is provided through the set of beryllium bronze "finger-springs" along the $120 \mathrm{~mm}$ long dissociator neck. The "finger-springs" conventional application is to provide flexible electrical contact in RF-systems. Similar springs are used in the ANKE and BLAST ABS instead of indium foil for heat transfer from the tip of the dissociator quartz liner. In this design, the whole dissociator neck is cooled by the cold head through "fingersprings", which allows to operate the dissociator neck at significantly lower temperature to reduce wall surface recombination and improve hydrogen atoms thermal equilibrium conditions. An aluminum nozzle is attached directly to this tube with an indium foil in between for better heat conductance. The nozzle diameter is $2.0 \mathrm{~mm}$. A diode temperature sensor is attached to the nozzle. The temperature difference between cold head and the nozzle is about $30^{\circ}$. The second strap (10-foils) cools the top stainless steel part of the dissociator neck at a temperature difference of about $100^{\circ}$. A second temperature sensor is attached to the top copper bracket. Two more diodes measure the cold head and the neck base temperatures. The temperature stabilization system includes two cylindrical heaters $(5 \mathrm{~mm}$ in diameter $50 \mathrm{~mm}$ long), which are mounted in the body of the cooling nozzle bracket. The dissociator and the first skimmer positions are designed to be adjustable without breaking vacuum. This allows a fine tuning of the nozzle-skimmer and skimmer-sextupole magnet gaps, which are critical for the optimal ABS performance. The optimal nozzle-skimmer gap is $18 \mathrm{~mm}$ and the skimmer-first sextupole gap is $22 \mathrm{~mm}$. The dissociator transverse alignment is produced by four adjustable rods (see Fig.2), which hold the long dissociator body in place and reduce vibration caused by the cold head.

A tunable master oscillator and a broadband amplifier $(0.2-35 \mathrm{MHz}$ range) are used to power the dissociator RF-cavity, which is similar to BLAST ABS design /7/. The RF matching for minimal reflected power is produced by frequency tuning, (typically the reflected power doesn't exceed 2\%). At present the dissociator is operated at 21.6 $\mathrm{MHz}$. The dissociator frequencies in the various operational ABS differ from 13.56 $\mathrm{MHz}$ to $100 \mathrm{MHz}$, but there are no systematic experimental studies of optimal frequency value. With the broadband RF system we plan to study dissociator performance at different frequencies in range $13.6-35.0 \mathrm{MHz}$ and higher, if necessary. Higher frequency microwave dissociators at $2.5 \mathrm{GHz}$ are based on a different, surfacewave technique of RF-power input to the dissociator plasma discharge. Very promising results of degree of dissociation increase at high gas flow were obtained at the HERMES ABS, in particular for the atomic deuteron beam $/ 8 /$.

The sextupole separating magnet system. The separating magnet system was designed to maximize the atomic beam density in the collision region. It consists of six permanent sextupole magnets (manufactured by Vacuumschmelze, Germany), which are divided in two groups. The 1-4 magnets separate the atomic beam by electron spin projection and the 5-6 magnets focus the beam to the collision chamber center about $320 \mathrm{~mm}$ downstream of the last magnet. The magnet system geometry is shown in Fig.3. A distinctive feature of this design is a $65 \mathrm{~cm}$ long drift space between 
separating and focusing magnets. The $32 \mathrm{~cm}$ chamber $\# 4$ can be replaced with a shorter $20 \mathrm{~cm}$ chamber to study the effect of the drift space length on the ABS performance. Other conditions being equal, the long drift space allows to reduce the gas load to the collision chamber. The magnets are assembled in two holders, which are aligned to the chamber centers. The measured atomic beam position at the collision point is just 0.7 $\mathrm{mm}$ off the chamber center, which was achieved without any additional optical alignment just due to precisely manufactured vacuum chambers. The calculated optimal atomic beam velocity is about $2.0 \cdot 10^{5} \mathrm{~cm} / \mathrm{sec}$, which can be obtained with the dissociator nozzle temperature cooled to $80^{\circ} \mathrm{K} / 3 /$.

RF-transitions. Two identical sets of a strong field transition (SFT) and a weak field transition (WFT) are used in the ABS and BRP part of the H-jet setup. The RFtransitions are shielded from the longitudinal holding magnet field by additional magnetic screens. RF-transition efficiencies of $99.9 \pm 0.1 \%$ were measured for both weak- and strong-field transitions with the BRP polarimeter.

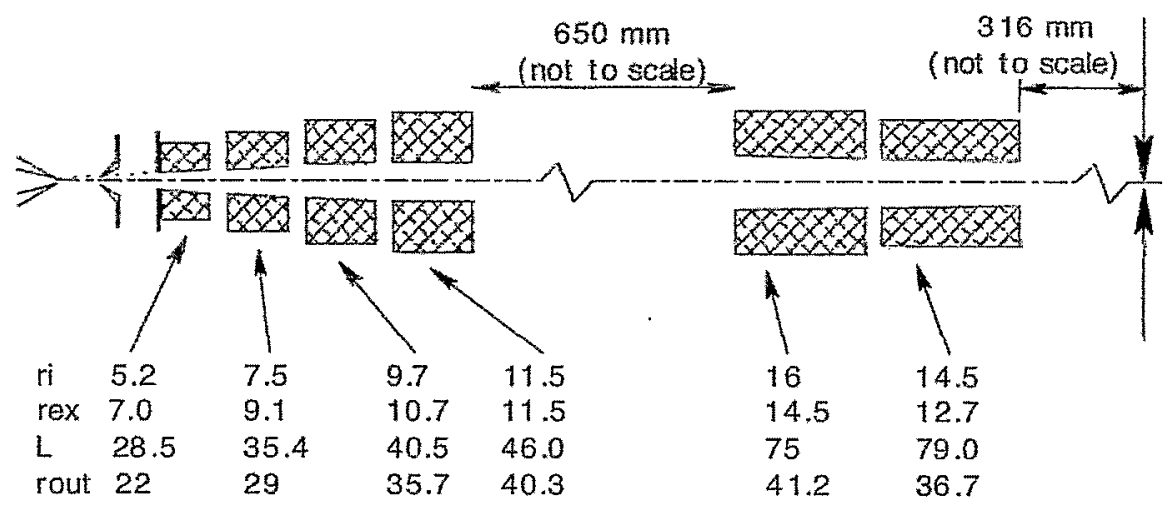

Fig.3. Separating magnet system geometry. The entrance and exit radii, overall length, and outer radius of each magnet are indicated (all dimensions are in $\mathrm{mm}$ ).

\section{b). SCATTERING CHAMBER.}

The CNI elastic p-p collision asymmetry is peaked at a momentum transfer I $\mathrm{t} 1 \sim 0.001-0.02(\mathrm{GeV} / \mathrm{c})^{2}$, which corresponds to the recoil proton scattering angles 85 89 degrees for RHIC beam energies of $25-250 \mathrm{GeV} / 2 /$. The silicon strip recoil detectors are situated at $80 \mathrm{~cm}$ distance from the jet-target in the recoil-arm cylindrical chambers, which are attached to the standard central chamber (see Fig.4). The scattering angle is defined by the collision point within $\sim 10 \mathrm{~mm} \mathrm{H}$-jet and the position sensitive silicon detector. The recoil proton energy is defined from the silicon detectors and the time-of-flight is used to suppress the background. The scattering angle can be further constrained by remotely adjustable collimators located at $\sim 4.0 \mathrm{~cm}$ distance from the target.

At the intersection point colliding bunches arrive at the same time and cannot be resolved by TOF. Therefore, the two beams will be separated spatially in the horizontal plane by about $10 \mathrm{~mm}$, and the entire polarimeter apparatus can be scanned across the 
two beams in horizontal direction. In this way scattering for one beam at a time can be selected in the polarimeter target, since the $\mathrm{H}$-jet size is less than $10 \mathrm{~mm}$.

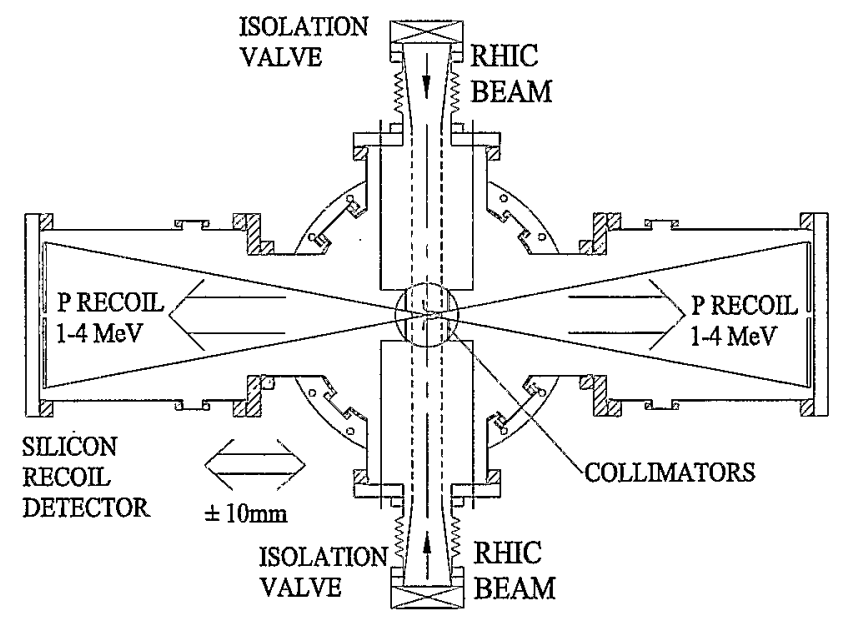

Figure 4. Scattering chamber. The distance from collision point to silicon detector is $80 \mathrm{~cm}$.

Holding field magnet: The $\mathrm{H}$-jet proton polarization vertical direction in the collision region is determined by the holding field magnet. The coils and magnetic steel plates of this magnet are enclosed in stainless steel casings, which are sealed in between standard vacuum chambers, so that high-current vacuum feedthroughs are not required. The holding magnet steering effect to the recoil protons is minimized by using compensation coils, whose fields are adjusted to keep the total vertical field integral along the proton paths close to zero. The maximum field in the collision region is 1.4 $\mathrm{kG}$, which gives the maximum proton polarization $\sim 97 \%$. A magnetic field homogeneity better than $10^{-3}$ within $\sim 4.0 \mathrm{~cm}$ center region was measured, which should allow to avoid depolarization by tuning the holding field in-between the depolarizing resonant transitions in the atomic beam, induced by the bunch field of the RHIC beam.

\section{ATOMIC BEAM INTENSITY AND PROFILE MEASUREMENTS}

The atomic beam profile was measured with a $2 \mathrm{~mm}$ in diameter compression tube. At the center of the collision chamber the FWHM of the beam is $5.5 \mathrm{~mm}$ (see Fig.5). The total beam intensity in the collision chamber was measured in two different ways. The first method utilized a compression tube of $10 \mathrm{~mm}$ in diameter and $100 \mathrm{~mm}$ length. The compression tube was calibrated by the conventional technique (pressure drop in calibrated, hydrogen filled volume) and independently by using MKS mass-flow controller (1 sccm total range). The measurement scheme (Fig.6) permits the calibrated hydrogen flow to be supplied either to compression tube or to chamber \#7, next to the collision chamber. A maximum atomic beam intensity of a $(12.4 \pm 0.2) \cdot 10^{16}$ atoms $/ \mathrm{sec}$ was measured at $\sim 75 \mathrm{sccm}$ flow, dissociator RF-power $280 \mathrm{~W}$ and nozzle temperature of $70^{\circ} \mathrm{K}$ (see Fig.7,8). In the second method the beam passed through an $18 \mathrm{~mm}$ collimator at the exit of the chamber \#6, and was dumped into chamber \#7 and subsequently pumped at $2000 \mathrm{l} / \mathrm{s}$ (BRP isolation valve was closed). The pressure 
increase produced by the beam was compared with the increase from calibrated flow, similar to a compression tube measurement. This measurement helps to exclude the possible systematic errors caused by the different gage sensitivities to atomic and molecular hydrogen. In the compression tube and large vacuum chamber the recombination and equilibrium conditions are quite different, also a hot cathode gage is used in compression tube measurements and the cold cathode gage in the \#7 chamber. Nevertheless, the measurements agree within $5 \%$.

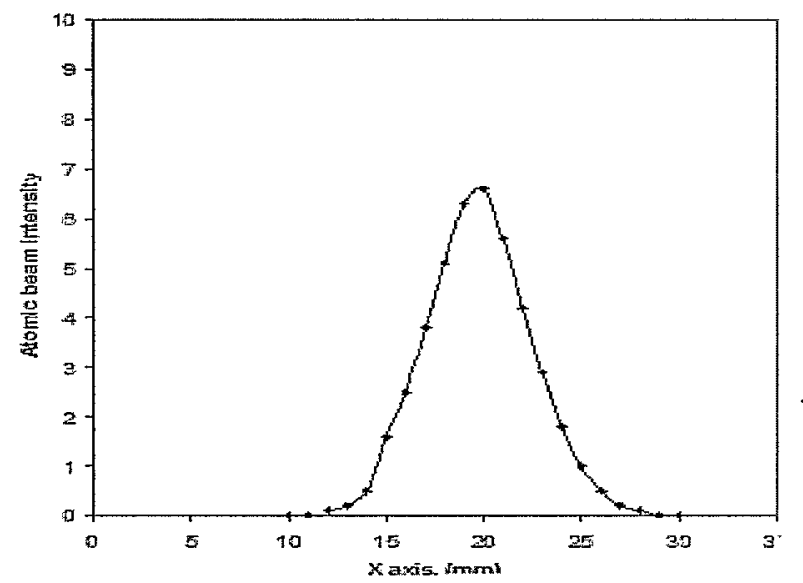

Fig.5. The measured atomic beam profile at the collision point. The FWHM, corrected for the finite size of the compression tube aperture, is $5.5 \mathrm{~mm}$ ).

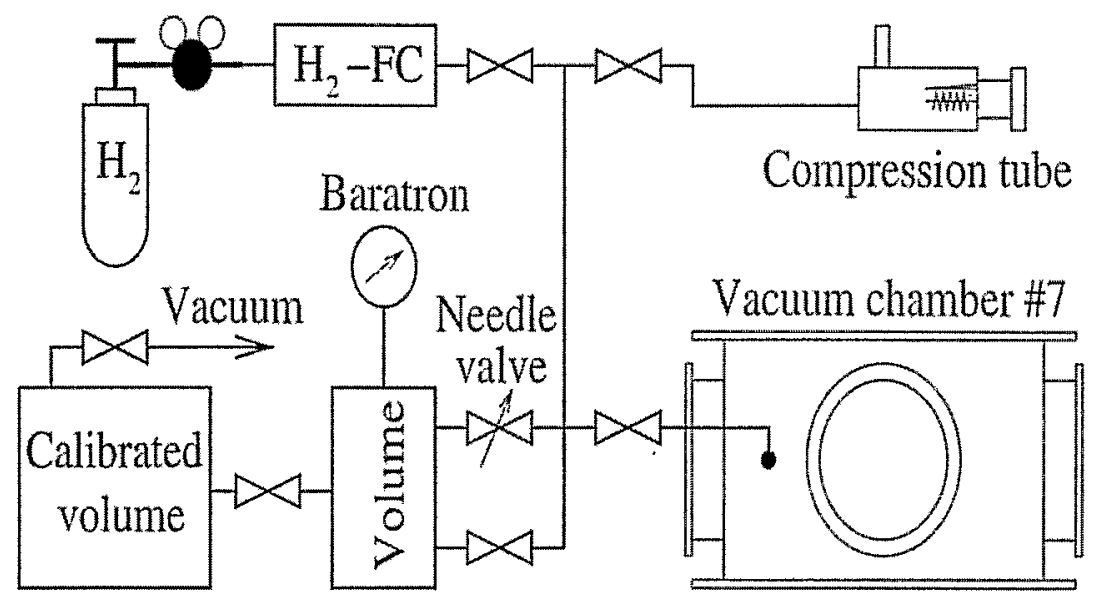

Fig.6. Calibration scheme for atomic beam intensity measurements. H2-FC- MKS mass flow controller. 


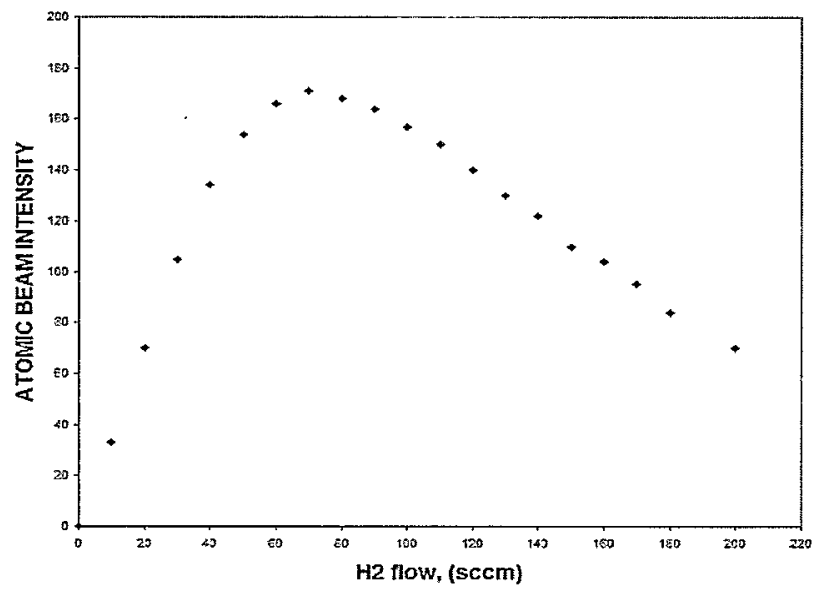

Fig.7. Atomic beam intensity vs. $\mathrm{H}_{2}$ flow in dissociator. The maximum $12.4 \cdot 10^{16}$ atoms/s intensity was obtained at $75 \mathrm{sccm} \mathrm{H}_{2}$ flow.

As a further cross-check, the beam intensity was also estimated from the known TMP pumping speed (a $1000 / \mathrm{sec}$ pumping speed for hydrogen is specified by manufacturer and measured by us in a separate tests). An intensity of a $(13 \pm 1) \cdot 10^{16}$ atoms/sec was calculated at this assumption, which is agrees well with the other measurements.

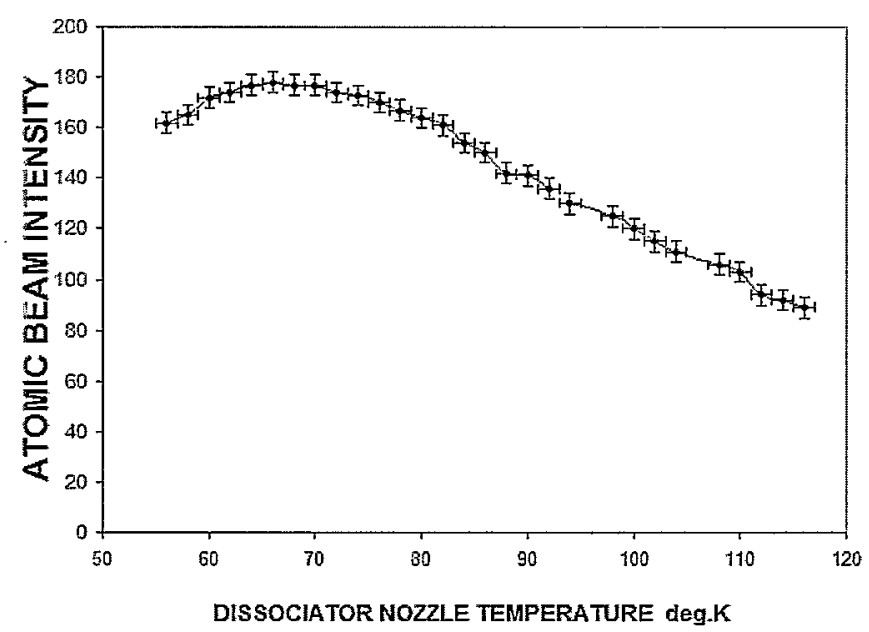

Fig.8. Atomic beam intensity vs. dissociator nozzle temperature.

\section{H-JET POLARIZATION MEASUREMENTS}

The proton polarization of the atomic beam was measured to $\sim 0.1 \%$ accuracy by taking a set of atomic beam intensity measurements at different RF-transition settings. To derive the effective target proton polarization, as seen by the RHIC beams, from BRP measurements a number of systematic error contributions have to be determined with high accuracy. 
Beam induced depolarization: The effect of bunched-beam induced resonance depolarization can be directly measured in the BRP. This depolarization is not uniform across the target cross-section because the accelerated beam size of $\sim 1.0 \mathrm{~mm}$ in diameter is much smaller than the jet-target. The BRP samples about $30 \%$ of the atomic beam, and the correction error might be large even if depolarization is just a few percent. To avoid this problem, the plan is to suppress the depolarization resonances by tuning the strength of the holding field in-between adjacent resonances. The resonance strength as a function of the holding field value will be measured using the BRP.

The target polarization dilution by molecular hydrogen and water vapor: The molecular hydrogen contamination of the atomic beam can be at a few percents level. The target polarization dilution by the molecular hydrogen (or water vapor) is the main systematic error contribution to the polarization measurements. Detailed studies of polarization dilution will be required to reduce the contamination to the lowest possible level and to develop techniques for precision correction measurements to meet the polarimeter accuracy design goal.

\section{FUTURE PLANS}

The polarimeter construction and tests reported here were carried out at the $\mathrm{H}$-jet test facility in the linac injector complex. The polarimeter will be moved to the RHIC tunnel just before the polarized proton run and moved out for further development at the test facility. The polarimeter is designed to be movable, i.e. it can be taken apart and reinstalled in either place in 2-3 days. The first $\mathrm{H}$-jet polarimeter operation in RHIC is planned for the 2004 polarized run. The goals for the first run will be obtaining reliable operation at the designed intensity and high (over 95\%) atomic beam polarization and the RHIC $100 \mathrm{GeV}$ beam polarization measurement to about $10 \%$ absolute accuracy.

Addendum. On October 8, 2003 the H-jet polarimeter was moved and installed into the RHIC ring at IP-12 intersection. The $50 \mathrm{~m}$ long cable extensions were completed and tested. The H-jet operation was fully tested including precision RF-transition efficiencies measurements with the VME based data acquisition system. Vacuum in the ring of a $2 \cdot 10^{-8}$ mbar was achieved with the $\mathrm{H}$-jet operation at nominal $10^{17}$ atoms $/ \mathrm{sec}$ polarized beam intensity. The polarimeter was moved back to the test facility on November 15.

\section{ACKNOWLEDGEMENTS}

The authors are thankful to F.Rathmann and D.Eversheim for useful discussion and drawings of the ANKE and EDDA dissociators. We like to thank H.Kolster for advice on dissociator RF-cavity design and providing the RF-cavity prototype, which is still used in the H-jet after some modifications. We would like to thank G.Bunce, T.Roser, J.Alessi for strong support and useful suggestions. We appreciate T.Russo, K.Unger and A.Weston contributions to the $\mathrm{H}$-jet polarimeter control system development. 


\section{REFERENCES}

1. Akchurin N., et al., « Polarimetry for high energy polarized proton colliders with a jet target $»$, Proc.12 $2^{\text {th }}$ Int.Symp. on high-energy Spin Physics 1996, World Scientific, ed by C.W.De Jager , p.810, 1997.

2. Bravar A., "The Absolute polarimeter for RHIC", SPIN 2002, AIP Conf. Proc. 675, p.830, (2003).

3. Wise T., et al., "Design of a polarized atomic $\mathrm{H}$ source for a jet target at RHIC", SPIN2002, AIP Conf. Proc. 675, p.934, (2003).

4. Zelenski A.,et al., "Polarized $\mathrm{H}$-jet polarimeter for absolute polarization measurements in RHIC", SPIN 2002, AIP Conf. Proc. 675, p.954, (2003).

5. Baumgarten C. et al.., "The Hermes Breit-Rabi polarimeter", NIM A482,p.606, 2002. 6. Jinnouchi O.et al, "RHIC pC CNI polarimeter", AIP Conf. Proc. 675, p.817, (2003).

7. Kolster H., "The BLAST polarized target", Proc. PST 2001, World Scientific, ed. V.Derenchuk, p.37, (2002).

8. N.Koch, E.Steffens, Rev.Sci,Instr., 70(3), p.1631, (1999). 